\title{
Un marco de referencia para las enseñanzas artísticas visuales en dispositivos móviles
}

\section{A Framework for Visual Art Education in Mobile Devices}

\author{
Alfredo José Ramón Verdú \\ Universidad de Murcia. Murcia. España \\ alfredoramon@um.es \\ Alfredo Cuervo Pando \\ Universidad de Murcia. Murcia. España \\ acuervo@um.es \\ María Gracia Ruiz Llamas \\ Universidad de Murcia. Murcia. España \\ llamas@um.es
}

\begin{abstract}
Resumen
La irrupción de los dispositivos móviles en el proceso de enseñanzaaprendizaje en educación acaecida en los últimos años, ha permeado en todos los sectores educativos, y consecuentemente, ha requerido la incorporación en cada disciplina de nuevas metodologías de trabajo, o la adecuación de las existentes. En todos los casos, la incorporación de esta tecnología está demandando la concreción de estructuras organizativas capaces de adaptase a las potencialidades inherentes de los propios dispositivos móviles, y a los diversos enfoques conceptuales del proceso de enseñanza-aprendizaje.

En el presente trabajo se propone un modelo de estructura para las Enseñanzas Artísticas Visuales que integra las distintas fases y actuaciones necesarias para llevarlo a cabo, manteniendo al alumnado como eje central del proceso. El modelo se fundamenta por un lado, en la definición del escenario de aprendizaje, de la planificación estratégica, y de la fase operativa, y por otro lado en los contextos y los espacios de trabajo desde los que organizar las formas de intervención en las Enseñanzas Artísticas Visuales. Como resultado, el diseño de la instrucción de los dispositivos móviles, determina la planificación de las acciones en espacios de aprendizaje autónomo y auténtico, en los que la educación artística visual se desenvuelve. Finalmente se plantea la definición del prototipo, prueba, evaluación, e implementación, de la estrategia en el momento de producción.
\end{abstract}

\section{Palabras clave}

Educación superior, m-learning, comunicación interpersonal, innovación didáctica, estrategias educativas, educación artística, diseño de la instrucción, cibermedios.

\footnotetext{
Abstract

The entry of mobile devices into the teaching-learning process in education occurred during the last years, has permeated all educational sectors, and has consequently required the incorporation in every discipline of new working methodologies or the adjustment of the existing ones. In any event,
} 
RED. Revista de Educación a Distancia. Núm. 59, Artíc. 7, 30-04-2019

DOI: http://dx.doi.org/10.6018/red/59/07

the incorporation of this kind of technology is demanding the implementation of organizational structures capable of adapting to the inherent potentialities of mobile devices, as well as to the different conceptual approaches in the teaching-learning process.

In this work we propose a model of structure for the Visual Arts Education, which integrates the different phases and the necessary actions to be carried out, while considering learners as the central axis of the process. This model is based, on the one hand, on the definition of the learning setting, the strategic planning and the operational phase, and on the other hand, on the contexts and the working spaces from where the forms of intervention in the Visual Arts Education may be organised. As a final result, the instructional design of the mobile devices determines the planning of the actions in spaces of autonomous and authentic learning in which Visual Arts Education takes place. Finally, we propose the definition of the prototype, the test, the evaluation and the implementation of the strategy at the moment of production.

\section{Keywords}

Higher education, m-learning, interpersonal communication, didactic innovation, educational strategies, art education, instructional design, cybermedia.

\section{Introducción}

La relación entre las teorías de aprendizaje que guían los distintos enfoques epistemológicos de la enseñanza, y los modelos de enseñanza-aprendizaje del Mobile Learning (m-Learning), se tienen que materializar a través de la creación de estructuras que hagan realidad este proceso. Esta estructuración de métodos y contenidos con fines pedagógicos, denominado Diseño de la Instrucción o Diseño Instruccional (DI), ha adquirido un renovado impulso dentro del contexto tecnológico, lo que obliga a tratarlo desde la perspectiva del DI como proceso, paso previo a su puesta en práctica en entornos específicos de aprendizaje, con sistemas digitales como mediadores.

Las Enseñanzas Artísticas Visuales (EAV) han evolucionado desde conceptos tradicionales de investigación, basados en "...la universalidad científica, la relación causa-efecto, el funcionalismo mecanicista, y el método hipotético deductivo" (Efland, 2003, p. 81), hacia enfoques vinculados con las problemáticas que afectan a las relaciones sociales en todo su ámbito. El cambio que los dispositivos móviles han supuesto para la educación se relaciona más con la diversidad de enfoques, de escenarios, y de diálogos que pueden proporcionar o generar, que con una visión unidireccional de la enseñanza, lo que abre un campo real al aprendizaje personalizado frente a las estructuras monolíticas basadas en los contenidos, tal y como expresan Watson, Watson y Reigeluth (2013). En este sentido Eisner (2002) ya reivindicaba el papel activo del estudiante, al igual que Christensen, Horn y Johnson (2011), con el enfoque alumno-céntrico de la enseñanza, como elemento indispensable para que se dé la motivación en un contexto de aprendizaje en la era de la información.

Al-Hnaiyyan, Al-Sharhan, y Alhajri $(2017$, p. 2) establecen como a piedra angular del aprendizaje a distancia a la estrategia transformadora de las aulas inteligentes, ya que esta "...se enfoca en conectar la generación digital, mejorar las oportunidades de

Un Marco de referencia para la Enseñanzas Artísticas Visuales en dispositivos Móviles.

Alfredo José Ramón-Verdú, Alfredo Cuervo-Pando, María Gracia Ruiz-llamas. Página 2 de 24 
aprendizaje individualizadas, fomentar la innovación en el aprendizaje, mejorar la pedagogía digital de los docentes y lograr los mejores resultados posibles de las escuelas, como también una inversión en TIC". En este marco, las EAV implican un proceso de aprendizaje muy dinámico que no se ocupa únicamente de la producción artística, sino que este proceso es el mecanismo para "crear nuestras vidas expandiendo nuestra conciencia, dar forma a nuestras disposiciones, satisfacer nuestra búsqueda de significado estableciendo contacto con otros, y compartiendo cultura" (Eisner 2002, p. 3). Así, la creación artística visual en un marco social concreto, se dará en parte cuando el estudiante dé respuesta a sus inquietudes dentro de unas condiciones medioambientales particulares. La tecnología móvil, en este caso, se presenta como un medio a través del que integrar la información que proviene de los contextos, para experimentarla y compartirla junto a la que el alumno sea capaz de crear, reduciendo la distancia existente entre diversos estratos y grupos sociales, diferentes economías, reduciendo el tiempo y el espacio, y democratizando el acceso a la información, sin tener en cuenta edades ni géneros (Yuktirat, Sindhuphak, Kiddee, 2018).

De esta forma, la elaboración de propuestas de actuación educativa para las EAV mediante dispositivos móviles -en adelante EAVm-Learning- debería incorporar los componentes del aprendizaje que permitan la interacción con los contextos, con otras personas, y entre estas y los propios dispositivos móviles. Así, en este escrito se plantea como finalidad la definición de un marco teórico de referencia, dirigido de forma específica hacia las enseñanzas artísticas visuales, abordándolo desde la necesidad de comunicación e interacción social que plantea la creación artística, durante el proceso creativo en un contexto de aprendizaje dialógico, mientras se ejecutan acciones y procesos cognitivos en espacios reales y virtuales, en un marco de una interacción triple entre las dimensiones humana, tecnológica, y contextual.

\section{Contextos de aprendizaje en el EAVm-Learning}

Las EAV, dentro de un enfoque posmoderno, se consideran generadoras de conocimiento crítico sensible a los contextos sociales, valiéndose del acto creativo y comunicativo. La manipulación de materiales, el uso de las técnicas, y de los métodos artísticos, requieren de una conceptualización, de un trabajo analítico, y del conocimiento de la materia, que no parte únicamente de la intuición o de la capacidad creativa del individuo. Además de esta capacidad, intervienen los conocimientos, aptitudes, intereses, y acciones en el medio o contexto, en el que se desarrolla la actividad de aprendizaje. La movilidad que subyace en la tecnología móvil permite realizar nuevos enfoques de enseñanza, que como ya indicaba Freedman (2003), representan los contextos desde los que obtener experiencia, tanto por la observación y el análisis, como por la experiencia activa y directa, estableciéndose así la primera dimensión en el marco EAVm-Learning.

Los contextos de producción artística adquieren importancia por tratarse del medio natural de aprendizaje desde donde se extraen los significados para la producción artística. De manera que, para la utilización de dispositivos móviles en este aprendizaje, se deben tener presente varios aspectos: por un lado los contextos y los

Un Marco de referencia para la Enseñanzas Artísticas Visuales en dispositivos Móviles.

Alfredo José Ramón-Verdú, Alfredo Cuervo-Pando, María Gracia Ruiz-llamas. Página 3 de 24 
RED. Revista de Educación a Distancia. Núm. 59, Artíc. 7, 30-04-2019

DOI: http://dx.doi.org/10.6018/red/59/07

factores medioambientales, por otro, la comunicación que la propia tecnología permite y a la vez condiciona, y por último, los espacios comunicativos y de diálogo dentro de un paradigma educativo basado en el constructivismo social, a través de los medios digitales adoptados socialmente (Filali, Khalidi y Bennani, 2017; Tinashe y Cilliers, 2019). El alumno podrá, como indican Naismith, Lonsdale, Vavoula y Sharples (2004), construir en cada momento, bajo el apoyo y tutorización del profesor, la transferencia de un conocimiento de carácter conceptual/teórico, hacia esquemas prácticos de aprendizaje.

\section{Escenarios de aprendizaje para EAVm-Learning}

En las EAV se proporcionan escenarios en donde se trabajan los proyectos que determinan, en gran medida, la estrategia que el alumno seguirá de forma autónoma. En 1991 Lave y Wenger propusieron el paradigma del Situated Learning, o Aprendizaje Situado, en el que se afirma que no se puede entender el aprendizaje como algo aislado compuesto por factores y personas sin relación alguna; el aprendizaje se produce dentro de un contexto social específico - social y material-. A este hecho Lave y Wenger lo denominaron "participación periférica legítima". Aquí, el aprendiz se implica activamente en los momentos $y$ en los lugares donde se produce la circunstancia de conocimiento, dentro de actividades socio-culturales y/o profesionales, actividades en las que estos profesionales actuarían con el rol de facilitadores.

En el ámbito de las EAV también se integra el Action Learning, o Aprendizaje Activo, relacionado con la Teoría de la Actividad. Esta teoría se fundamenta en la Zona de Desarrollo Próximo (ZDP) planteada por Vygotsky (1978), aunque en una fase posterior evolucionó a lo que se considera una tercera generación, desarrollada por Engeström (1987). La ZDP se define como “...la distancia entre el nivel real de desarrollo, determinado por la resolución de problemas independientemente, y el nivel de desarrollo potencial, determinado a través de la resolución de problemas bajo la guía de un adulto o en colaboración con compañeros más capaces" (Vygotsky, 1978, p. 86), o lo que es lo mismo, el aprendizaje que puede realizar una persona por sí misma partiendo de su propio conocimiento e iniciativa, junto con el que adquiera a través de la orientación de un profesor o a través de la interacción con otras personas. Aquí se avanza en el aprendizaje, dejando a un lado el puramente cognitivista, para incluir un aprendizaje que se deriva de la interacción y la experiencia con otras personas y contextos, que bien podrían ser comunidades de aprendizaje, o aprendizaje mediado a través de dispositivos electrónicos (Kaptelinin y Nardi, 2006).

Así, la Teoría de la Actividad queda relaciona con el aprendizaje que se produce en la interacción con otras personas, y en una visión actualizada de los términos, con la interacción dentro de un contexto de aprendizaje mientras se ejecutan acciones, ya sea en espacios físicos o en espacios virtuales, como resultado de la interacción humana con el medio. Aquí reside la relación entre la Teoría de la Actividad y el mLearning. Esta teoría define el contexto como un sistema de actividades en el que la persona realiza su actividad nutriéndose de la información que le aporta el contexto, transformándola y devolviéndola procesada en forma de nuevas cuestiones o acciones.

Un Marco de referencia para la Enseñanzas Artísticas Visuales en dispositivos Móviles.

Alfredo José Ramón-Verdú, Alfredo Cuervo-Pando, María Gracia Ruiz-llamas. Página 4 de 24 
RED. Revista de Educación a Distancia. Núm. 59, Artíc. 7, 30-04-2019

DOI: http://dx.doi.org/10.6018/red/59/07

El aprendizaje es, por lo tanto, un proceso dialéctico interactivo en el que se realizan acciones, tareas, y operaciones, y que generan a su vez correspondencias con los motivos que propician las actividades, las metas a conseguir con las acciones, y las condiciones instrumentales para que las operaciones se lleven a cabo (Engeström, 1999).

El Authentic Learning, o Aprendizaje Auténtico, es otro de los conceptos que hace referencia al Aprendizaje Situado. Según Santos (2009) este es un proceso de doble dirección: situaciones y conocimientos sobre situaciones reales trabajadas dentro del aula, para después exportarlas fuera, en situaciones reales de aplicación. En estas circunstancias, las situaciones reales de la vida adquieren relevancia por el significado auténtico que poseen dentro de un paradigma de constructivismo social. Traxler (2009) define el Aprendizaje Auténtico de este modo:

Por Aprendizaje Auténtico nos referimos al aprendizaje que implica problemas y proyectos que son relevantes e interesantes para el alumno en el mundo real. Aprendizaje auténtico implica que el aprendizaje debe ser en torno a tareas auténticas, que los estudiantes deben participar en la exploración y la investigación, que los estudiantes deben tener oportunidades para el discurso social, y que amplios recursos deben estar disponibles para los estudiantes ya que persiguen problemas significativos (Traxler, 2009, p. 18).

Introduciendo la autenticidad en las metodologías docentes se incorpora a esta ecuación el momento y la circunstancia en la que se produce la actividad, permitiendo al aprendiz incorporar a su propio conocimiento esa circunstancia experimentada, favoreciendo así la creación de puentes cognitivos para la memoria a largo plazo (Morrow y Holland, 2011), o para la generación de juicio sobre lo que es fiable y no, la capacidad de síntesis, y la flexibilidad en diversos contextos desconocidos (Lombardi, 2007).

\section{Referentes de DI para EAVm-Learning}

Las EAV presentan una complejidad particular por tratarse de expresiones basadas en procesos creativos individuales, expresados a través de un filtro personal. La tecnología ofrece mecanismos para estructurar la información de forma conveniente para estos fines, lo que se traduce en la práctica, en la posibilidad de utilizar métodos y herramientas distintas a las tradicionales, abriéndose el campo a nuevas experiencias. Para cumplir este objetivo hay que determinar las partes que tiene que integrar el Diseño Instruccional (DI), para que el alumno ejerza un dominio del aprendizaje real en los niveles de comprensión, asimilación de significado, y dominio de la tarea, que permita realizar la construcción de jerarquías conceptuales durante el proceso de actividad.

El DI parte del trabajo que se tiene que realizar desde el lado del profesor/tutor, en el proceso de planificación y estructuración de los métodos de enseñanza, junto con otros especialistas necesarios para poner en funcionamiento un sistema digital complejo. Este trabajo se materializa en la actividad que el alumno desempeña en la

Un Marco de referencia para la Enseñanzas Artísticas Visuales en dispositivos Móviles.

Alfredo José Ramón-Verdú, Alfredo Cuervo-Pando, María Gracia Ruiz-llamas. Página 5 de 24 
búsqueda de las respuestas que todo proceso de aprendizaje implica, y que en última instancia, dentro de las EAV, dependerá en gran medida de los espacios, los contextos, y de la versatilidad que los sistemas $m$-Learning posean para beneficiar de forma específica a cada alumno.

El marco de referencia que se propone, y que se puede ver en la Figura 4, aprovecha aspectos del modelo de DI de Morrison, Ross, Kalman y Kemp (2013), así como el modelo de Prototipado Rápido de Tripp y Bichelmeyer (1990), desde la adaptación de Botturi, Canotoni, Lepori y Tardini (2007), y además con un enfoque constructivista, en su variante de constructivismo social planteado por Jonnasen (1999).

El modelo de Morrison et al. (2013) mencionado (Figura 1), se estructura en base a círculos concéntricos, orientado hacia el estudiante, y en el que cobra una especial importancia el análisis que se haga de él desde diversas direcciones: características generales, competencias específicas que poseen los alumnos, estilos de aprendizaje que presentan, formación previa que poseen para establecer un punto de partida, características sociales y personales de su entorno, diversidades culturales, inconvenientes o impedimentos que se pudiesen presentar como incapacidades, y las diferencias de edades dentro de un mismo grupo.

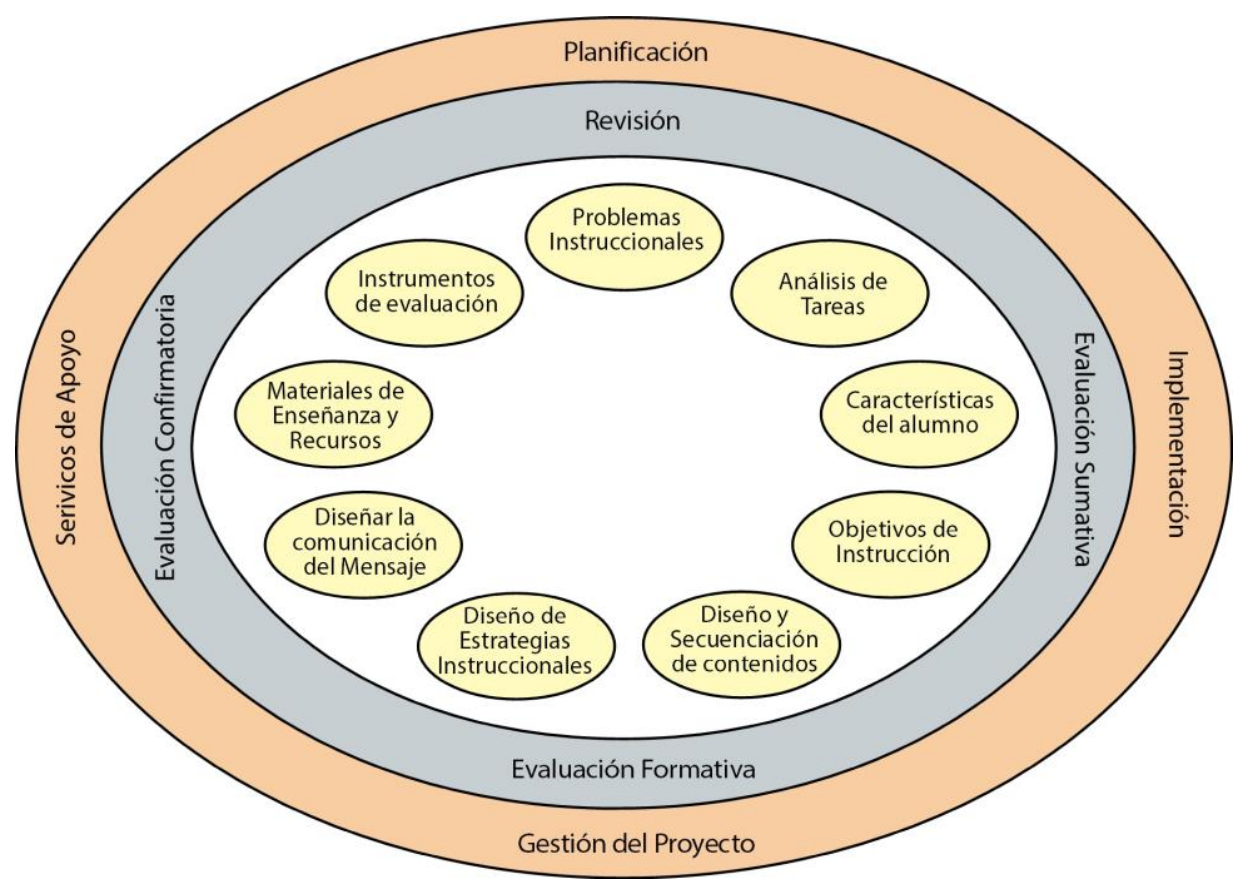

Figura 1. Modelo oval de Morrison, Ross, Kalman y Kemp (2013).

El modelo posee un enfoque que facilita la participación multidisciplinar, reforzado por el hecho de su modularidad, y la independencia de los elementos. Uno de los aspectos más interesantes del modelo de Morrison et al. (2013) radica en el concepto de versatilidad que ofrece al diseñador instruccional para introducir cambios y mejoras durante todo el proceso de elaboración y de ejecución, adaptándose a las necesidades 
que demanden los alumnos, ya que posee un carácter holístico de la enseñanza. Esta son características muy importantes para EAVm-Learning, es decir, la posibilidad de realizar intervenciones multidisciplinares sin tener que hacer adaptaciones en la estructura diseñada, ya que las EAV poseen aspectos difíciles de predecir y controlar, y esta versatilidad le hace ser valioso para estos fines.

El segundo, el modelo de Prototipado Rápido en la variante de Botturi et al. (2007), el cual se puede ver en la Figura 2, posee un enfoque más flexible que el anterior por permitir adaptaciones a los posibles enfoques micro de la enseñanza - lo que significaría cierto grado de especificidad en el proceso enseñanza-aprendizaje, evitando los "grandes relatos"-, centrando la atención sobre casos específicos. El modelo se diseñó para ser utilizado en sistemas digitales de enseñanza como alternativa plausible a grandes sistemas de DI con altos costes de desarrollo, es decir, dada la versatilidad que posee, hace posible que la adaptación dinámica que requieren las EAV pueda hacerse sin grandes desarrollos y estudios nuevos.

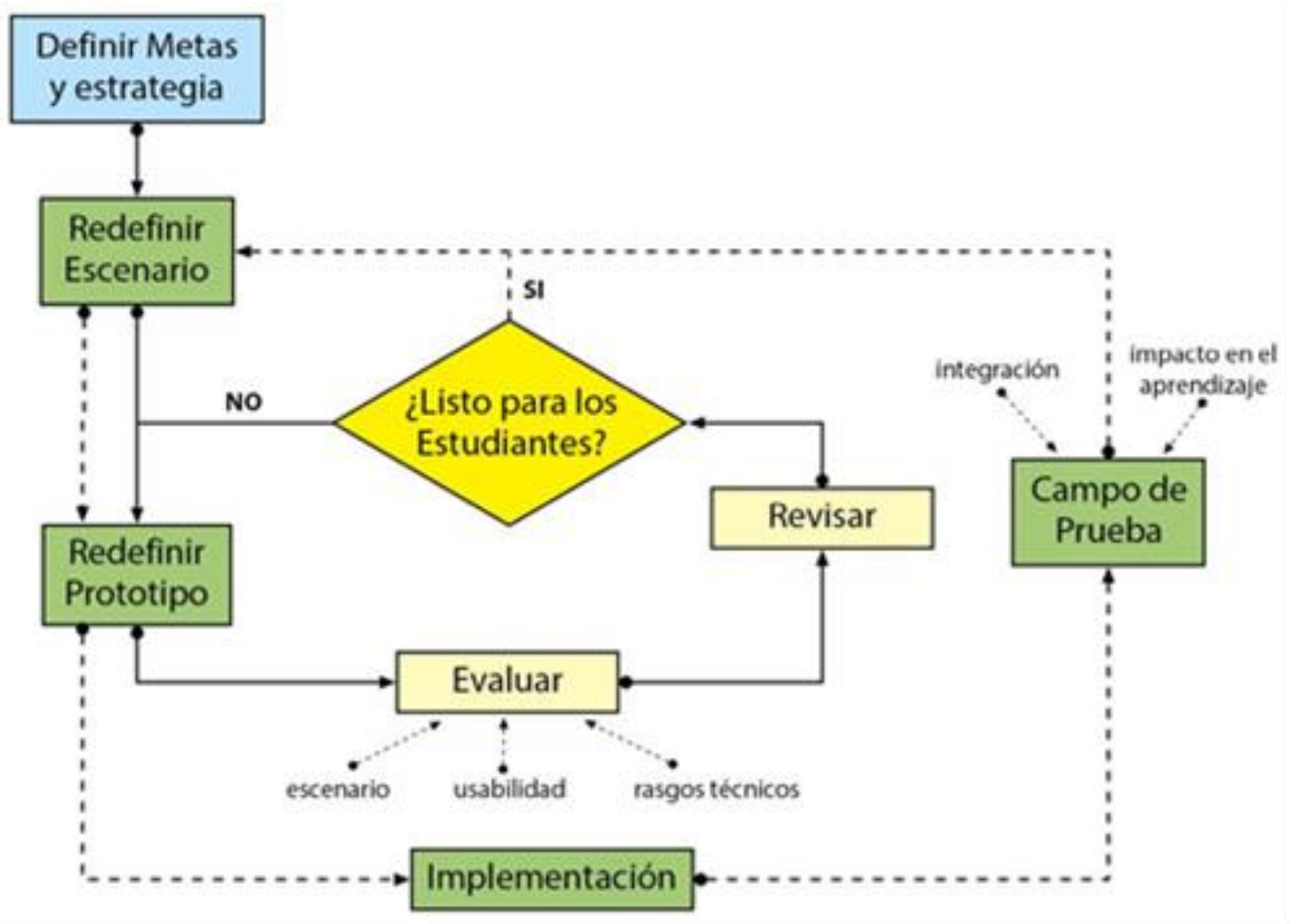

Figura 2. Variante de Prototipado Rápido de Botturi et al. (2007).

Para un aprendizaje basado en dispositivos móviles, tanto el contexto social como los marcos de aprendizaje, forman parte de una concepción constructivista (Jonassen, 1999). El modelo de Jonassen (Figura 3) se desarrolla mediante un trabajo continuo, dinámico y revisable. Se irán adaptando a los requerimientos contextuales y a los nuevos conocimientos que vayan surgiendo, por lo que no es un proceso lineal y secuencial. La fortaleza radica en que se trabaja partiendo de la idea de no linealidad de los hechos, y de la adaptabilidad. 


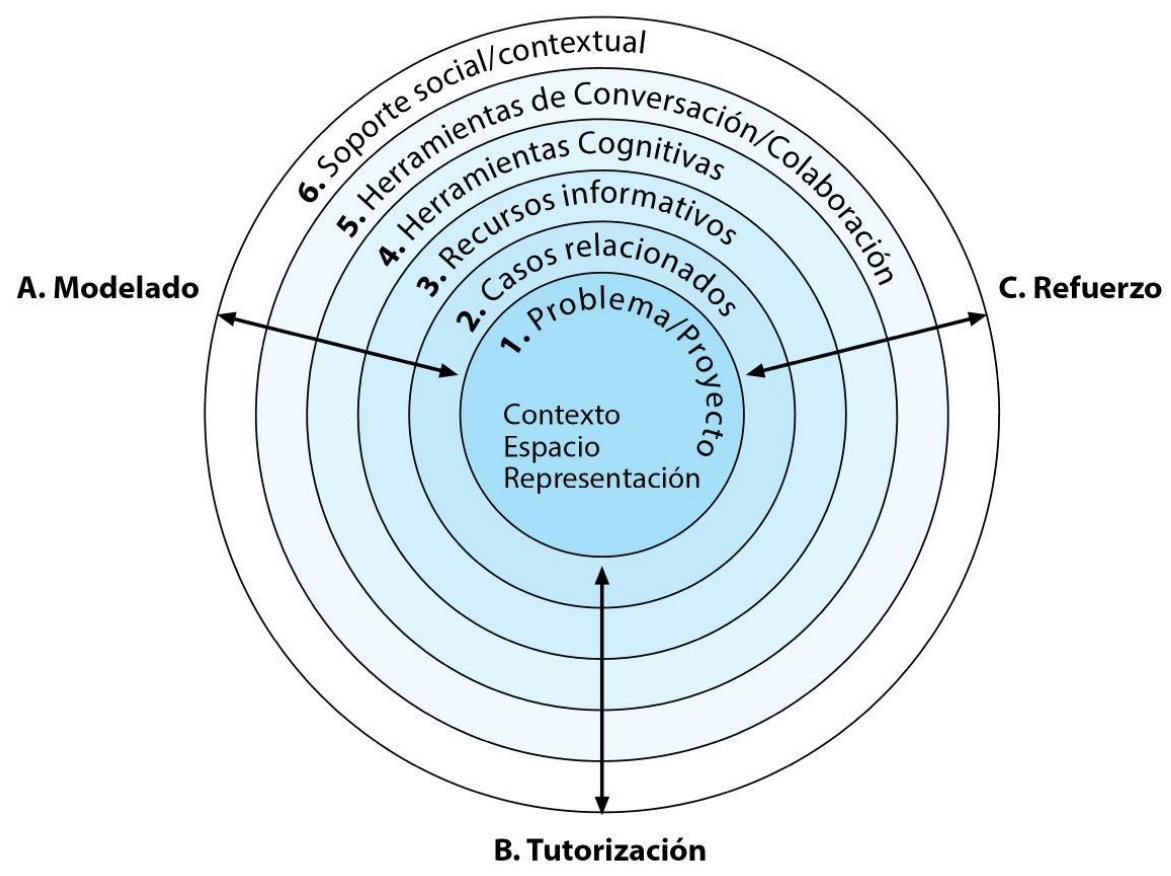

Figura 3. Adaptación del modelo de diseño de entornos constructivistas de Jonassen (1999).

En este modelo, las principales técnicas que se pueden utilizar son el Aprendizaje Basado en Problemas (ABP) y el Análisis o Estudio de Casos, de manera que se relacione la práctica con la vida real. Para definir un problema será necesario contextualizarlo en el ámbito de su desarrollo natural, definir el espacio en el cual se trabajará, y la representación y/o manipulación del problema en una situación real, pudiendo realizar así una aproximación cognitiva de los sucesos en un contexto dado, o que tenga un significado para los alumnos (Jonassen, 2000).

\section{Modelo Organizativo de DI para EAVm-Learning}

El m-Learning se caracteriza, entre otras cosas, por la movilidad, el tipo de comunicación que proporciona, y los espacios de trabajo en contextos reales que puede proveer, tal y como ya se ha comentado. Este es un aspecto fundamental para las EAV, en donde los contextos son el punto de partida del modelo EAVm-Learning. El modelo que se propone en la Figura 4 está compuesto por dos bloques principales, formados a su vez por varios círculos interiores. La lectura de estos círculos comienza desde el interior hacia el exterior y de izquierda a derecha. La propia estructura visual de círculos a modo de tronco de árbol, ayuda a crear un esquema mental de crecimiento por introducción de nuevos conceptos y estructuras exteriores, quedando la primera en el interior a modo de sustento. 
RED. Revista de Educación a Distancia. Núm. 59, Artíc. 7, 30-04-2019

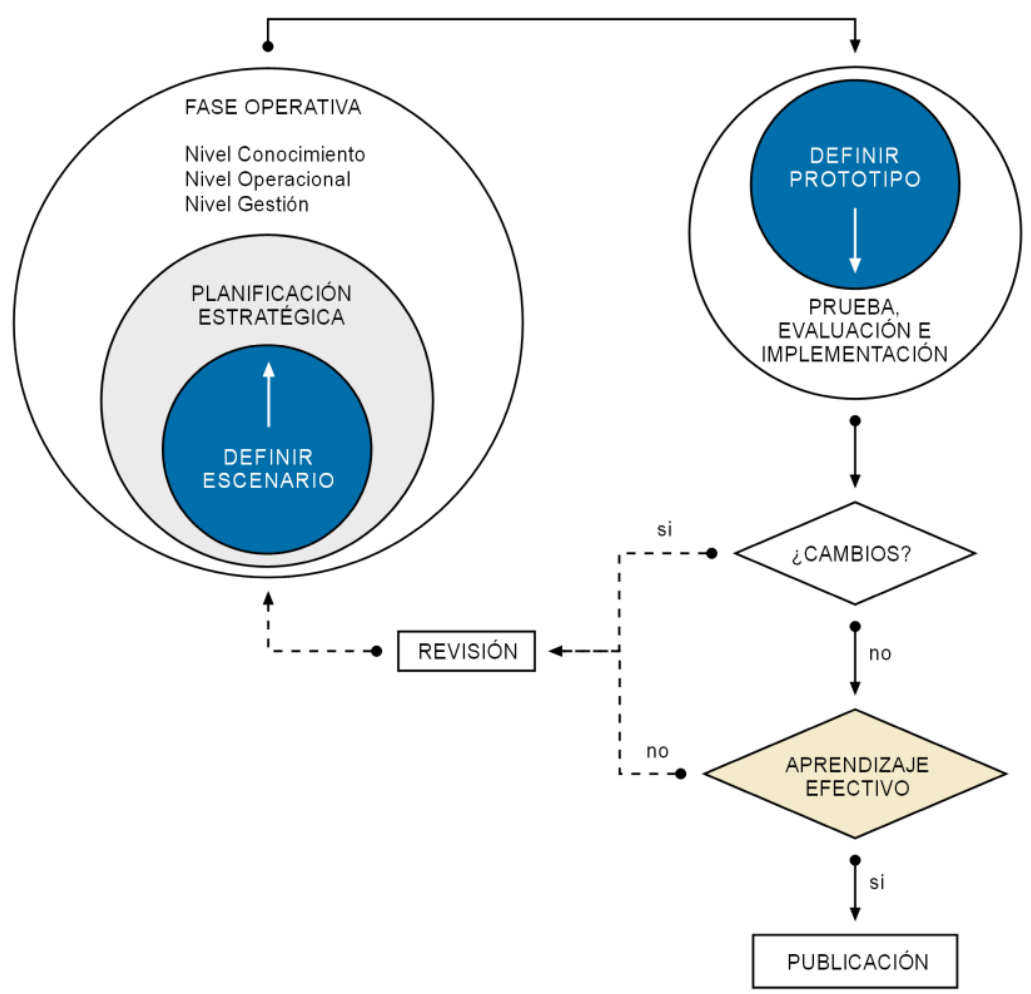

Figura 4. Modelo de DI para las EAVm-Learning

La zona de la izquierda corresponde a la fase de análisis y planificación. Consta de tres círculos excéntricos, siendo el primero que hay que leer el que corresponde a la Definición del Escenario, para pasar a la Planificación de la Estrategia, y a la Fase Operativa, en la que se definirán los niveles de intervención desde tres dimensiones: nivel conocimiento, operacional, y de gestión, dimensiones que se describirán más adelante.

Una vez definidos todos los aspectos que determinan esta primera etapa se continúa con la estructura de la derecha - de producción y evaluación-, que como la anterior, su lectura será de dentro hacia fuera, por lo que la primera etapa será la Definición del Prototipo, para pasar a su Prueba, Evaluación e Implementación definitiva.

La distinción en dos grupos denominados análisis y planificación, y producción y evaluación (Figura 5) está fundamentada por pertenecer a dos momentos diferenciados del proceso. La utilización de círculos excéntricos fortalece además la percepción del modelo como un proceso dinámico en progresivo crecimiento, contrapuesto a esquemas estáticos y monolíticos. 


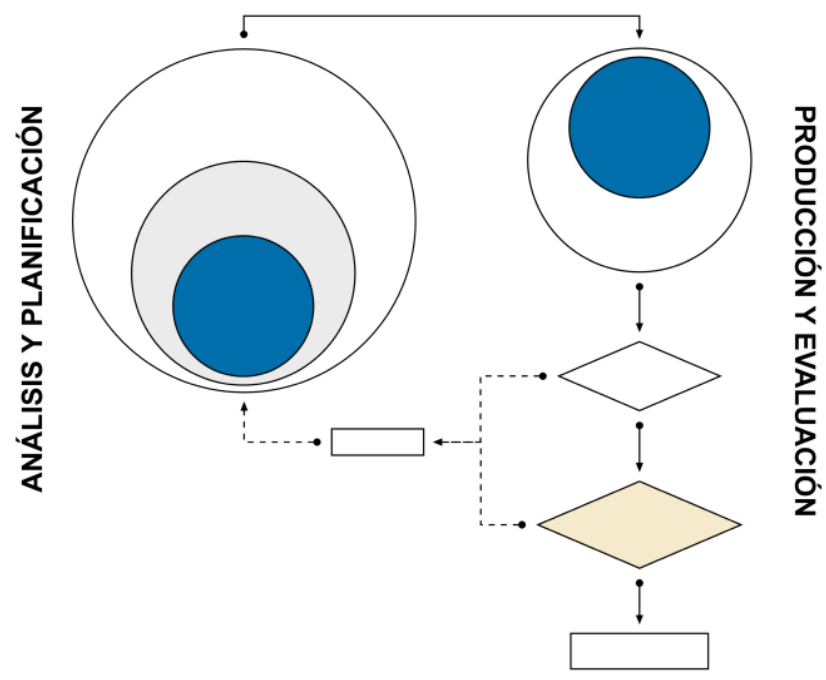

Figura 5. Momentos del modelo EAVm-Learning

\subsection{Definición del Escenario de Aprendizaje}

En el modelo EAVm-Learning se propone como punto de partida la definición del escenario, ya que desde las EAV, la comprensión y análisis de la realidad que subyace en las temáticas relacionadas con la identidad, el discurso de género, las desigualdades o los procesos personales internos, entre otros, son las materias que constituyen el trabajo sobre proyectos.

Entendiendo el aprendizaje como un proceso, la evolución personal es consustancial al aprendizaje, al igual que la transformación y la transferencia de resultados, que también irán creciendo en calidad y en cantidad según transcurra el proceso. Por tanto, el análisis inicial del alumnado se debe entender como un proceso variable y no como una magnitud fija. Lo mismo debe ocurrir con los materiales y con los recursos, que deben ir adaptándose a la propia evolución del alumno y a su demanda, dada la especial idiosincrasia de las EAV.

En el escenario se determina la estrategia más adecuada y los objetivos que se trabajarán. Aquí se analiza al estudiante de EAV, el contexto real en donde se tiene que producir el aprendizaje, y los factores que van a estar presentes en el proceso. Así, dentro del EAVm-Learning, el primer aspecto a analizar es el espacial desde el punto de vista de la autonomía del alumno:

1. El aprendizaje en espacios controlados, que habitualmente se produce en las aulas y en zonas de talleres artísticos, y en los que suele haber una presencia constante del tutor.

2. El aprendizaje en espacios autónomos incontrolados, en los que el alumno decide qué aspectos formativos son los que le interesa en cada momento, dentro de las temáticas y las formas de trabajar que se le propongan de forma individual o grupal. 
RED. Revista de Educación a Distancia. Núm. 59, Artíc. 7, 30-04-2019

DOI: http://dx.doi.org/10.6018/red/59/07

En el análisis de los alumnos hay que determinar los aspectos educativos que ya poseen y cuáles será necesario potenciar, teniendo en cuenta los estilos de aprendizaje, sus intereses, la formación que poseen, o su capacidad técnica, entre otros.

Para autores como Smaldino, Russell, Heinich y Molenda (2004) son tres las categorías desde las que analizar al alumno, donde los tópicos que hemos mencionado anteriormente están integrados:

- Características generales.

- Competencias específicas.

- Estilos de aprendizaje.

Morrison et al. (2013) amplían estas categorías con:

- Información académica.

- Características sociales y personales.

- Diversidad cultural.

- Estudiantes con discapacidad.

- Estudiantes adultos.

De los datos que se deriven del análisis de los alumnos en los tópicos mencionados, se tendrá que extraer el perfil del alumnado, cuáles serán sus potencialidades y limitaciones, y, por lo tanto, permitirá definir una estrategia educativa adaptada a las características que posean los escenarios de trabajo.

Otro aspecto del análisis del escenario se tiene que referir a la obtención de datos físicos de los espacios, tales como: ubicación, iluminación, equipamiento disponible, relevancia, limitaciones, etc., lo que supone analizar los requerimientos técnicos, espaciales y temporales de los que se dispone, o que son necesarios. Lo que sucederá en estos contextos dependerá del nivel de instrucción que se haya dispuesto, del nivel formativo, y del nivel de implicación de los alumnos (Morrison et al., 2013).

Para Koole (2006), el m-Learning se produce en contextos de información en el que están integrados los aspectos del estudiante, los sociales que rodean el marco de aprendizaje y los tecnológicos que actuarán como medio. De forma específica para las EAV, el análisis del contexto se tiene que observar desde tres enfoques distintos: el contexto como proveedor de información, el contexto como medio o espacio de trabajo sobre el que se interviene, y una tercera relacionada con el diálogo interactivo que se produce al realizar una intervención directa, ya que generará nueva información que puede volver a formar parte de la propia propuesta artística. El contexto aquí no se presenta como algo estático, sino que podría generar nueva información mediante la intervención y la actividad artística situada.

Un Marco de referencia para la Enseñanzas Artísticas Visuales en dispositivos Móviles.

Alfredo José Ramón-Verdú, Alfredo Cuervo-Pando, María Gracia Ruiz-llamas. Página 11 de 24 
RED. Revista de Educación a Distancia. Núm. 59, Artíc. 7, 30-04-2019

DOI: http://dx.doi.org/10.6018/red/59/07

\subsection{Planificación estratégica}

Esta planificación supone el plan maestro en donde se define el tipo de aprendizaje que se llevará a cabo y cómo es posible ejecutarlo. La definición de un enfoque estratégico para el EAVm-Learning debe girar en torno a las distintas metodologías y modalidades de enseñanza posibles. Ha de ser consistente y adaptativa a la variedad de contextos posibles, organizando los elementos espaciales, humanos y técnicos, e incluso aspectos emocionales que se pudieran dar (Reigeluth et al., 2015). En esta cobrará forma el verdadero diálogo entre los componentes que participan en el proceso de aprendizaje en un contexto dado, y que mediante la tecnología móvil se hará efectivo (Figura 5).

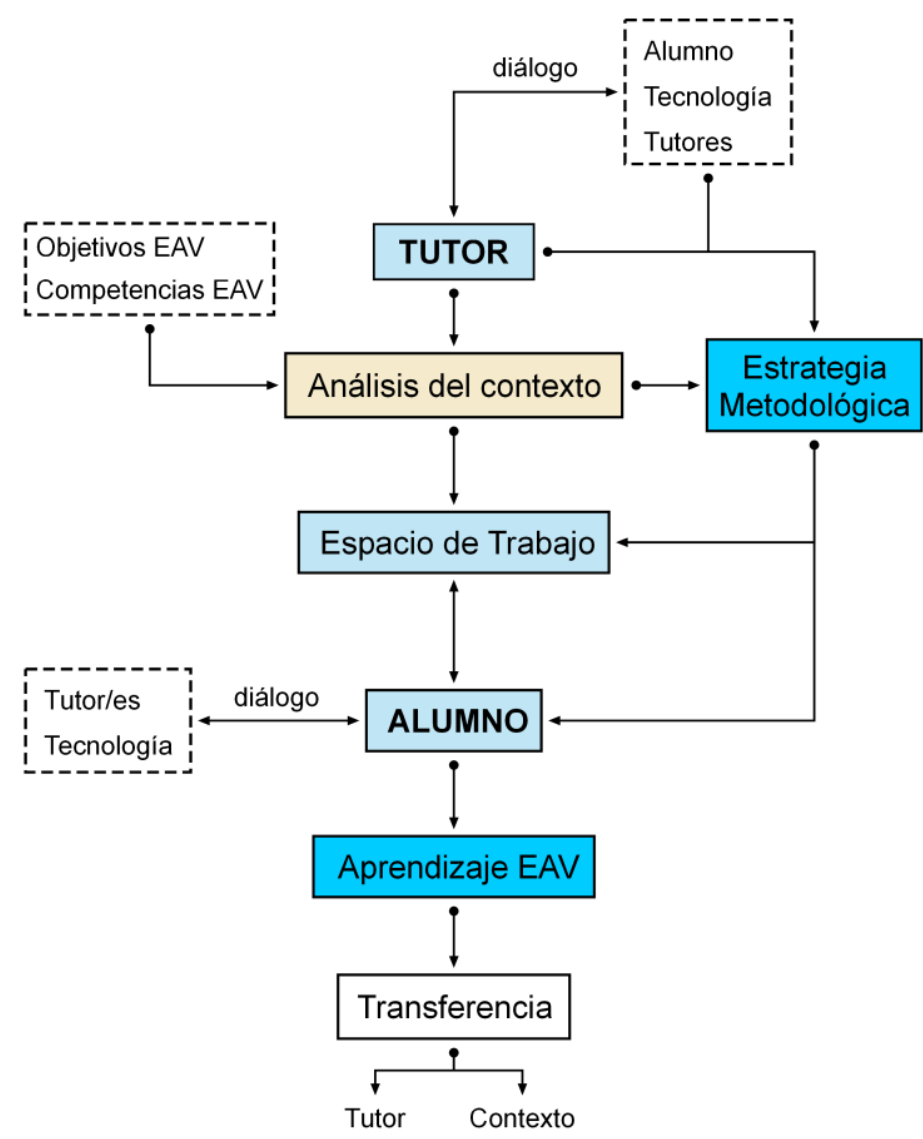

Figura 6. Diagrama general de planificación estratégica para EAVm-Learning.

Dada la importancia que tiene el contexto y el aprendizaje auténtico en el m-Learning (Buchem y Camacho, 2011; Herrington y Herrington, 2007; Traxler, 2009; Wang y Shen, 2012), la estructura general de planificación estratégica comienza con el reconocimiento por parte del tutor de ese contexto como espacio plausible de contener elementos útiles para un aprendizaje EAVm-Learning. Las interacciones que se realicen (Figura 6) dependerán del tipo de metodología utilizada, independientemente de la autonomía que el alumno posea (Moore, 1993; Park, 2011).

Un Marco de referencia para la Enseñanzas Artísticas Visuales en dispositivos Móviles.

Alfredo José Ramón-Verdú, Alfredo Cuervo-Pando, María Gracia Ruiz-llamas. Página 12 de 24 
RED. Revista de Educación a Distancia. Núm. 59, Artíc. 7, 30-04-2019

DOI: http://dx.doi.org/10.6018/red/59/07

Las relaciones de diálogo - autónomas o guiadas - evidencian este diálogo como hilo conductor del aprendizaje.

El siguiente elemento principal de esta planificación estratégica es la Estrategia Metodológica que se lleve a cabo. En este sentido habría que plantearse cuál es la mejor manera de afrontar la enseñanza de las artes visuales, pregunta que supone el gran reto de la enseñanza en todos los ámbitos educativos. Puesto que esto dependerá de todos los factores que afectan al contexto educativo, no tiene una respuesta unívoca, aunque sí podría tener respuestas parciales a situaciones concretas. En las EAV se potencia el aprendizaje profundo, el cual se produce cuando se pone en marcha la comprensión, desde un punto de vista holístico, de las circunstancias del aprendizaje, que incluye el pensamiento crítico, el creativo, y el complejo, lo que nos lleva a mencionar a Morrison (2007), por sostener que para que esto se produzca es preciso habilitar un entorno de aprendizaje constructivista -en su vertiente social para el m-Learning-, en el que se den los marcos que van a contribuir a la creación de un aprendizaje profundo y holístico en entornos colaborativos, dinámicos y adaptables, y que además sea well facilitated, lo que determinará la estructura y la entrega de materiales, propuestas, enfoques, etc., a las que el alumno habrá de enfrentarse.

\subsubsection{Navegación Operativa en la Estrategia Metodológica}

El uso de la tecnología móvil se realiza a través de los interfaces que permiten la comunicación del humano con la máquina. Por lo tanto la interacción de una máquina con la persona depende de la composición estructural física de los aparatos, y de los elementos visuales que le acompañan. Así, la relación producida por el uso particular del dispositivo está muy relacionada con su operatividad, la capacidad de personalización que disponga, el placer intrínseco en su uso, y el feedback que proporcione. Es por ello que el diseño de interfaces tiene que proporcionar la navegación y la estructura sobre la que discurra el aprendizaje de forma agradable, motivadora y en circunstancias auténticas.

Para EAVm-Learning la navegación operativa se basa principalmente en los planteamientos expresados en la Teoría del Conectivismo (Siemens, 2004), en la teoría del Navigacionismo (Brown, 2005), y en el modelo de enseñanza Conversacional de Laurillard $(2002,2007)$, todo ello desde la perspectiva del estudiante.

La navegación constituye uno de los mecanismos fundamentales sobre el que se construye el conocimiento en entornos digitales, permitiendo al alumno realizar su propio trayecto de aprendizaje mediante el discurrir entre los elementos que se le muestren dinámicamente. Él decidirá autónomamente, y con una visión holística, cuando ha de hacer uso de ellos y con qué propósito en relación a los problemas que se le planteen (Freedman, 2003).

Con este planteamiento, la enseñanza está ahora centrada en el proceso de aprendizaje, tal y como un enfoque posmoderno en las EAV plantearía, y no basada en los contenidos (Hernández 2010), forzando a que se produzca un

Un Marco de referencia para la Enseñanzas Artísticas Visuales en dispositivos Móviles.

Alfredo José Ramón-Verdú, Alfredo Cuervo-Pando, María Gracia Ruiz-llamas. Página 13 de 24 
cambio de rol del profesor actuando como facilitador del aprendizaje. Así, será preciso una participación activa del estudiante indagando y profundizando por sí mismo en los conceptos, creciendo en su propio proceso metacognitivo (Narciss, 2008).

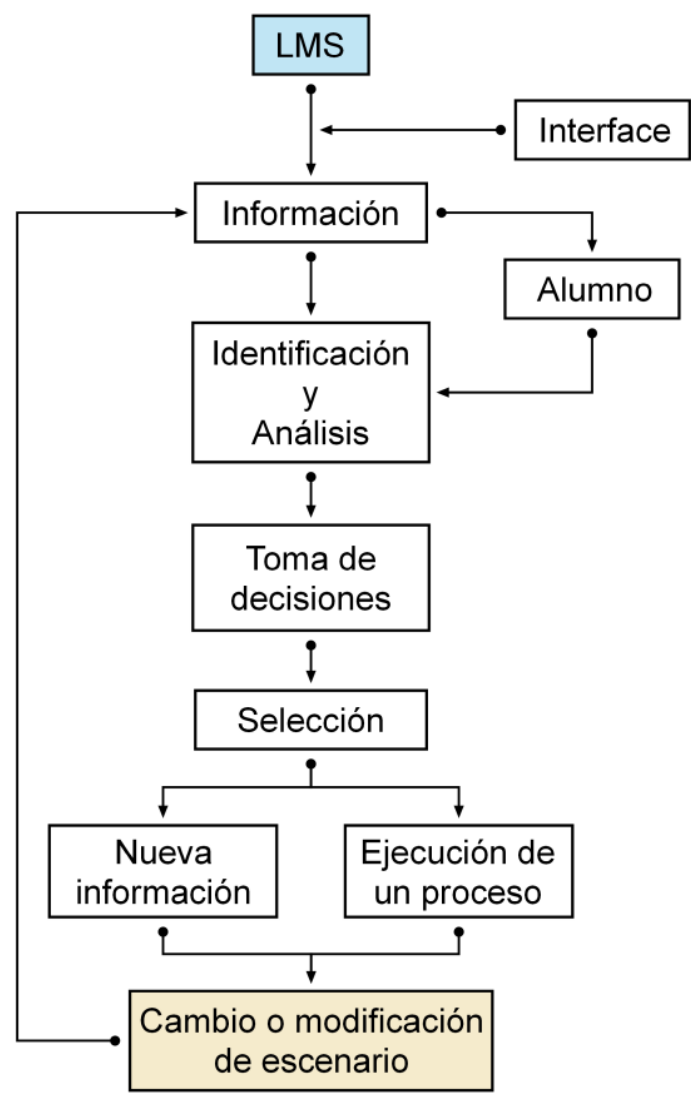

Figura 7. Diagrama sobre el proceso de navegación en EAVm-Learning.

Como se aprecia en la Figura 7, el proceso de selección dentro de un ambiente de navegación en EAVm-Learning se traducirá en un cambio o una modificación del escenario en donde volverá a entrar en juego nueva información. Esto significa que cualquier proceso que se ejecute dentro de un contexto móvil generará un nuevo espacio en el que el alumno tendrá que volver a realizar una nueva identificación y análisis de la información resultante, y tomar nuevas decisiones que volverán a determinar el flujo informativo. Esta navegación tiene que facilitar que el alumno pueda llevar a cabo una serie de procesos distintos, como por ejemplo el de establecer la conversación con el tutor o con el grupo de trabajo en espacios compartidos (Holmberg, 1995; Laurillard, 2007), permitir la creación de portafolios individuales dentro de los espacios personales, revisar los conocimientos previos así como los conceptos, ideas y preguntas de aprendizaje, analizar casos, permitir la profundización intelectual autónoma, recolectar datos, interactuar con el medio, recibir retroalimentación, autoevaluación, etc. 
RED. Revista de Educación a Distancia. Núm. 59, Artíc. 7, 30-04-2019

DOI: http://dx.doi.org/10.6018/red/59/07

\subsection{Fase operativa.}

Volviendo a la explicación de la Figura 4, lo que hemos denominado como Fase Operativa en el modelo EAVm-Learning, corresponde a la parte del DI que se ocupa de los aspectos relacionados con el desarrollo y la gestión del sistema digital. Aquí se establecen los roles de las personas y su ámbito de intervención, así como sus responsabilidades en la administración de los medios informáticos y/o educativos.

La Fase operativa posee tres niveles diferenciados: nivel de conocimiento, nivel operacional, y nivel de gestión. Se deben desarrollar simultáneamente ya que en algunos momentos las funciones se pueden solapar.

- Nivel Conocimiento: Este nivel abarca el ámbito del proceso enseñanzaaprendizaje en toda su globalidad. Está compuesto por el grupo de expertos, en este caso por especialistas en las materias, y será responsabilidad suya la de realizar el enfoque pedagógico de la enseñanza que integra la metodología general, los contenidos, los materiales necesarios, las competencias y objetivos, la evaluación, así como gestionar el nivel de profundidad que de forma individualizada cada alumno demande según su propia evolución.

- Nivel Operacional: El nivel operacional se refiere a las operaciones que hay que realizar de forma habitual dentro de un sistema de gestión de información digital. Por lo tanto hay que definir las intervenciones relacionadas tanto con la enseñanza, como con las modificaciones que la infraestructura técnica informática requiera: comunicaciones, respuestas a circunstancias particulares, rectificación de materiales, preparación de materiales nuevos, cancelación de actividades, adaptaciones hacia alumnos concretos, contacto con especialistas, preparación logística en desplazamientos, convocatorias a Webinars, etc.

- Nivel Gestión: El nivel de gestión se refiere por un lado a las labores de organización de la información por parte de los especialistas informáticos actuando como apoyo técnico, y por otro lado a las tareas administrativas que desde el Learning Management System (LMS) habrá que realizar para la implantación y mantenimiento del sistema educativo. Estos sistemas requieren ser instalados, optimizarlos, realizar revisiones, copias de seguridad, mantenimiento de las bases de datos, desarrollar y adaptar recursos y módulos que aporten nuevas características al sistema, dar soporte técnico, establecer los roles y los niveles de acceso de cada uno de las partes, integración de elementos gráficos, gestión de las analíticas del aprendizaje, etc.

Todos los modelos de DI que se han analizado distinguen en mayor o menor medida las dimensiones que se plantean en la Fase Operativa, aunque cada uno de ellos la expresa en distinto momento, y con enfoques diversos. Por ejemplo, a parte de los modelos mencionados (Botturi, et al., 2007; Morrison et al., 2013; Tripp y Bichelmeyer, 1990; Jonassen, 1999), el modelo ADDIE, como modelo de los más utilizados en entornos e-Learning, establece dentro de su dimensión de Diseño, el bosquejo de las unidades, lecciones, o módulos, así como también el diseño pedagógico, el diseño gráfico, el diseño de la evaluación, etc., aspectos incluidos en los niveles de

Un Marco de referencia para la Enseñanzas Artísticas Visuales en dispositivos Móviles.

Alfredo José Ramón-Verdú, Alfredo Cuervo-Pando, María Gracia Ruiz-llamas. Página 15 de 24 
Conocimiento y de Gestión de nuestra propuesta. En la fase de Desarrollo de ADDIE es donde crea la estructura informática, los elementos multimedia, o todo lo relacionado con el hardware y el software necesario, están incluidos en el nivel Operacional y en el de Gestión. Lo mismo ocurre con el modelo de Dick, Carey y Carey (2015), que después de realizar el desarrollo de la estrategia instruccional, contemplan la selección y generación de materiales, pero en este caso, y según bajo nuestro criterio, obviando por no ser esa su intencionalidad - el contexto como eje central de un planteamiento para m-Learning, cuestión que en nuestro enfoque si se tiene en cuenta, y del que deriva el resto de componentes del marco de referencia que se propone.

\subsection{Definición del prototipo}

La definición del prototipo significa poner a prueba transitoriamente todo lo propuesto en los apartados anteriores, desde la definición del escenario, pasando por la planificación estratégica, y la concreción de la fase operativa con los niveles que la conforman. La creación del prototipo supone poner en marcha todos los aspectos tratados para poder realizar una revisión, el control y el perfeccionamiento de las partes. Esto implica que cada elemento del grupo de trabajo ha realizado su tarea, y que esta se materializa en un sistema informático con capacidad operativa plena y que cumple una función, con una vida limitada por ser un prototipo, y para cubrir una finalidad muy concreta. Está compuesto por los aspectos técnicos, pedagógicos y contextuales. Su puesta en práctica para ser evaluado se ciñe a la verificación de los componentes que lo forma, organizados de manera coherente para proponer tareas y espacios de trabajo al alumno. Por lo tanto, cada tarea -enfoque micro de la enseñanza ya mencionado- implica el diseño de un prototipo distinto para ponerse en práctica temporalmente y evaluarlo antes de su implantación definitiva.



Figura 8. Ámbito del Prototipo en EAVm-Learning.

Un Marco de referencia para la Enseñanzas Artísticas Visuales en dispositivos Móviles.

Alfredo José Ramón-Verdú, Alfredo Cuervo-Pando, María Gracia Ruiz-llamas. Página 16 de 24 
RED. Revista de Educación a Distancia. Núm. 59, Artíc. 7, 30-04-2019

DOI: http://dx.doi.org/10.6018/red/59/07

Todos los componentes del prototipo han de ser funcionales, y el planteamiento conceptual de las tareas debe estar perfectamente preparado para que cumpla su función, así como también los elementos de diseño gráfico activos. La navegación ha de estar perfectamente operativa y proporcionar el soporte necesario para que el alumno trabaje autónomamente cuando así se requiera la tarea.

\subsection{Prueba, evaluación e implementación}

Esta fase del DI es donde se analiza el prototipo y se evalúa si el diseño creado proporciona los espacios de aprendizaje y los métodos correctamente organizados y expresados para que cumplan su función. Además se realiza la revisión y evaluación de cada una de las partes que lo compone, desde el punto de vista tecnológico, pedagógico, organizativo y de apoyo. Se pondrá en funcionamiento el planteamiento sobre casos reales, ejecutados por personas con distintos roles (tutor, alumno, especialista, técnico, encargado del sistema de apoyo, de infraestructura, diseño gráfico, etc.), para detectar fallas, incoherencias y posibles errores no contemplados anteriormente a nivel de programación informática, organizativo y pedagógico desde una evaluación formativa.

El carácter que se le ha asignado al modelo EAVm-Learning es el de un modelo flexible y dinámico en el que se podrían realizar cambios en tiempo real en el momento de detectar algún fallo en el planteamiento del tipo que sea, ya que estos cambios afectarán únicamente a las tareas. No ocurriría así con un cambio estructural o en uno tecnológico, que implicaría un mayor desarrollo y consumo de tiempo en la modificación.

Esta evaluación también hay que realizarla desde el rol del profesor como usuario, y desde la perspectiva del estudiante como persona destinataria de las actuaciones. También habrá que evaluar si los materiales docentes cumplen su función. Del mismo modo que también habrá que evaluar los espacios, los contextos, la navegabilidad y si el diseño gráfico.

En este proceso de creación e implementación del modelo, una vez realizados los pasos descritos, será necesario comprobar si el diseño efectuado proporciona un aprendizaje efectivo. Esta comprobación puede ofrecer un resultado positivo, con lo que resultaría la publicación definitiva del diseño realizado, o negativo, lo que implicaría una revisión cíclica de los componentes afectados.

\section{Secuencia básica en el marco EAVm-Learning}

Para la mejor comprensión del planteamiento teórico llevado a cabo, en la Tabla 1 se incluye una secuencia básica de actuación para la elaboración de una tarea desde el marco de referencia EAVm-Learning. La tarea mencionada se debe entender como cualquier acción que el alumno debe llevar a cabo, dentro del campo disciplinar, y que suponga una acción intelectual, manipulativa, o verbal, que suponga un grado de elaboración, por lo que aquí están integradas tareas del tipo de estudio de casos,

Un Marco de referencia para la Enseñanzas Artísticas Visuales en dispositivos Móviles.

Alfredo José Ramón-Verdú, Alfredo Cuervo-Pando, María Gracia Ruiz-llamas. Página 17 de 24 
RED. Revista de Educación a Distancia. Núm. 59, Artíc. 7, 30-04-2019

DOI: http://dx.doi.org/10.6018/red/59/07

resolución de ejercicios y problemas, aprendizaje basado en problemas, orientado a proyectos, o trabajo cooperativo.

Está organizada según las distintas dimensiones implicadas, y organizadas secuencialmente según la elaboración temporal que habría que realizar.

Tabla 1. Secuencia básica orientativa para EAVm-Learning

Dimensión Acciones

TAREA/PROBLEMA EAV

Definición de tareas, problemas, o Establecer la pertinencia respecto al programa curricular. casos

Objetivos de aprendizaje

DEFINICIÓN DE ESCENARIO

Características del estudiante

Análisis del contexto
Definición de los objetivos y competencias EAV implicados en la tarea, sobre los que el alumno trabajará.

Objetivación de las características sociales y personales, formación y conocimientos previos, diversidad cultural, discapacidades, potencialidades, etc.

Espacios y momentos de aprendizaje:

- Autónomos controlados: aulas y talleres.

- Autónomos incontrolados: intereses propios y de entorno social.

Características del espacio físico:

- Ubicación, iluminación, equipamiento, relevancia, limitaciones, ruido, temperatura, etc.

Características del espacio móvil:

- Acceso del alumno al espacio personal, al colectivo, al virtual, al tecnológico, al híbrido.

Información que provee el contexto para la tarea EAV:

- Espacial perceptivo: medio natural, museos virtuales o reales, espacios urbanos, exposiciones, etc.

- Como espacio de trabajo reflexivo: realización de fotografías, videos, grabación de conversaciones y sonido, toma de notas y muestras, análisis metodológico de obras y situaciones, realidad aumentada, etc.

- Como espacio de intervención: ejecución de proyectos personales de forma activa, auténtica y situada.

\section{PLANIFICACIÓN ESTRATÉGICA}

Objetivación del contexto

Ámbito de uso del espacio virtual móvil
Espacio plausible para la tarea EAV ya analizada en el análisis del contexto.

Almacenamiento de datos, aplicaciones de autor (App), bloc de notas, Bluetooth, calendario plataformas para compartir

Un Marco de referencia para la Enseñanzas Artísticas Visuales en dispositivos Móviles.

Alfredo José Ramón-Verdú, Alfredo Cuervo-Pando, María Gracia Ruiz-llamas. Página 18 de 24 
RED. Revista de Educación a Distancia. Núm. 59, Artíc. 7, 30-04-2019

DOI: http://dx.doi.org/10.6018/red/59/07

Ámbito de uso del espacio híbrido

Ámbitos de intervención y producción

Estrategia metodológica información, grupos de contactos y de trabajo, llamadas telefónicas, mensajería instantánea, radio, SMS (texto e imagen), USB, Wifi, etc.

Códigos QR (gráfico, textual e Internet), creación y edición fotografía, video, yincanas educativas, grabación de sonido, grabación de video, impresión directa, impresión 3D, información contextual (ej. SIRI de iPhone), mapas terrestres, posicionamiento Geográfico (GPS), proyección de imágenes, Realidad Aumentada, reconocimiento gestual y de voz, Realidad Virtual, video conferencia, MOOC, redes sociales, etc.

Definición de las temáticas, p. ej.: Las tradiciones visuales como crítica, grupos étnicos, la imagen como medio, contextos políticos y religiosos, el espacio urbano aglutinador de la diversidad cultural, contextos históricos, arquitectura y urbanismo, identidad personal, tecnología y sociedad, la forma, espontaneidad creativa, tecnología y arte, etc.

Definir metodología:

- Conductista, cognitivista, constructivista social, ABP, situado, contextual...

Definir la arquitectura informativa:

- Receptiva, directiva, descubrimiento guiado, exploratoria.

- Cómo navegará el alumno por los contenidos.

- Establecer las conexiones conceptuales entre elementos, materiales, técnicas, autores, procedimientos, etc.

- Prever la no linealidad del aprendizaje en EAV-mLearning: pueden darse distintos puntos de arranque y distintas rutas de aprendizaje sobre una misma tarea o proyecto.

Establecer cómo será el diálogo alumno-profesor, y en qué momento.

Definir el sistema de apoyo al alumno.

Establecer cómo será la evaluación, en qué momento y herramientas.

\section{DEFINICIÓN FASE OPERATIVA}

\section{Nivel conocimiento}

Nivel operacional

\section{Relacionado con la Estrategia Metodológica.}

Definir los especialistas que deben intervenir, y en qué momento.

Preparación de contenidos adaptado al m-Learning:

- Materiales audiovisuales, cuestionarios de evaluación, mapas conceptuales, presentaciones, videotutoriales, etc.

Definir los objetivos de aprendizaje implicados en cada caso y momento de la tarea, y la retroalimentación ligada a cada uno.

Definir qué, cómo, y cuando se evalúa.

Definir cómo se produce la entrega de materiales según la arquitectura informativa empleada, y cuando.

Definir cómo, quién, y cuando se realizarán las intervenciones relacionadas con la enseñanza y con el sistema digital 
RED. Revista de Educación a Distancia. Núm. 59, Artíc. 7, 30-04-2019

DOI: http://dx.doi.org/10.6018/red/59/07

planteado:

- Comunicaciones con los alumnos, respuestas a circunstancias particulares, rectificación de materiales, preparación de materiales nuevos, cancelación de actividades, adaptaciones hacia alumnos concretos, contacto con especialistas, preparación logística en desplazamientos, convocatorias a Webinars, etc.

- Administración de RRSS educativas y foros de especialistas (Whatsapp, Facebook, Google foros, Instagram, etc.), habilitadas para el curso o la tarea.

Nivel gestión

Determinar las necesidades, la organización, y la administración informática por especialistas informáticos y diseñadores, que hagan realidad el planteamiento educativo:

- Instalaciones informáticas, optimizaciones, revisiones, copias de seguridad, mantenimiento de las bases de datos, diseño gráfico, etc.

- Gestión informática del LMS, si existe.

DEFINICIÓN DEL PROTOTIPO

Tarea alumno-céntrica

- Revisión de aspectos técnicos.

- Revisión de aspectos pedagógicos.

- Revisión de aspectos contextuales.

PRUEBA, EVALUACIÓN E IMPLEMENTACIÓN

Test del prototipo

Evaluación positiva

Evaluación negativa

Implementación
Puesta en producción del prototipo (online), en difusión reducida.

Revisión y evaluación de cada una de las partes que lo compone, desde los aspectos:

- Tecnológico.

- Pedagógico.

- Organizativo.

- Apoyo.

- Las relaciones entre cada una de las partes.

Revisión desde dos dimensiones: rol profesor y rol alumno.

Se podría plantear y evaluar un MVP (Producto Mínimo Viable), dependiendo de la envergadura de la tarea. Determinado por los costes y por las personas implicadas.

Difusión general de la tarea, práctica, curso, o proyecto de intervención artística, a través de: enlaces, mensajes, QR, etc.

Identificación de la falla.

Comunicación con el responsable, y rectificación.

Subsanado: retorno al test del prototipo y evaluación.

Puesta en producción tras la evaluación positiva.

Un Marco de referencia para la Enseñanzas Artísticas Visuales en dispositivos Móviles. 
RED. Revista de Educación a Distancia. Núm. 59, Artíc. 7, 30-04-2019

DOI: http://dx.doi.org/10.6018/red/59/07

\section{Conclusiones}

La adaptación de las Enseñanzas Artísticas Visuales a procesos tecnológicos relacionados con los dispositivos móviles, habitualmente se centra en el uso de diversas aplicaciones móviles, utilizadas de forma aislada, y dirigidas principalmente hacia la distribución unidireccional de contenidos, o la realización de actividades individualmente. La propuesta que se ha expresado representa la estructura organizativa de un marco de referencia, que desde el punto de vista docente se debería abordar para llevar a cabo esta disciplina. Este planteamiento permite integrar diversos métodos docentes, teniendo como eje central los contextos de trabajo del alumno, integrando, además, los aspectos de gestión que requiere un planteamiento tecnológico de este tipo. Dada la importancia que posee el contexto para las EAV origen de la información, y destino de las producciones artísticas-, el proceso de DI tiene que partir del contexto y de la información que este aporta, así como de las posibilidades de interacción y de intervención que el medio ofrece, lo que conllevaría un aprendizaje auténtico y activo desde marcos sociales conectados con la realidad. En este marco, el modelo EAVm-Learning propuesto podría formar parte de una metodología más amplia en la que el aprendizaje del alumno, a través de los dispositivos móviles, y la producción artística que se lleve a cabo, por un lado se realice de forma tradicional, y por otro lado utilizando los dispositivos móviles como plataformas de aprendizaje, creación, y difusión de resultados de las investigaciones, estableciendo así diálogos interactivos y enriquecedores compartidos.

Presentación del artículo: 10 de enero de 2019

Fecha de aprobación: 9 de abril de 2019

Fecha de publicación: 30 de abril de 2019

Ramón-Verdú, A. J., Cuervo-Pando, A., y Ruiz-Llamas, M. G. (2019). Un Marco de Referencia para las Enseñanzas Artísticas Visuales en Dispositivos Móviles. RED. Revista de Educación a Distancia, 59. DOI http://dx.doi.org/10.6018/red/59/07

\section{Financiación}

Esta investigación no ha recibido ninguna subvención específica de los organismos de financiación en los sectores públicos, comerciales o sin fines de lucro.

\section{Referencias}

Al-Hnaiyyan, A., Al-Sharhan, A. y Alhajri, R. (2017). A New Mobile Learning Model in the Context of Smart Classroom Environment: A Holistic Approach. International Journal of Interactive Mobile Technologies (iJIM), 11(3). DOI: https://doi.org/10.3991/ijim.v11i3.6186

Un Marco de referencia para la Enseñanzas Artísticas Visuales en dispositivos Móviles.

Alfredo José Ramón-Verdú, Alfredo Cuervo-Pando, María Gracia Ruiz-llamas. Página 21 de 24 
RED. Revista de Educación a Distancia. Núm. 59, Artíc. 7, 30-04-2019

DOI: http://dx.doi.org/10.6018/red/59/07

Ally, M. (2004). Using learning theories to design instruction for mobile learning devices. mLearn 2004, 3rd world conference on m-learning (http://goo.gl/GOAmmh).

Botturi, L., Canotoni, L., Lepori, B. y Tardini, S. (2007). Fast prototyping as a communication catalyst for e-learning design. En M. Bullen y D. P. Janes (Eds.). Making the transition to e-learning. Strategies and Issues (pp. 266-283). Hershey: Idea Group Inc.

Brown, T. H. (2005). Beyond constructivism: Exploring future learning paradigms. Education Today, 2. New Zeland: Aries Publishing Company (http://goo.gl/u71QH4).

Buchem, LI. y Camacho, M. (2011). M-project: first steps to applying action research in designing a mobile learning course in higher education (http://goo.gl/YrNrgO).

Christensen, C., Horn, M. B. y Johnson, C. W. (2011). Disrupting Class: How Disruptive Innovation Will Change the Way the World Learns. New York: McGraw Hill.

Colón, B., Taylor, K. A., y Willis, J. (2000). Constructivist instructional design: Creating a multimedia package for teaching critical qualitative research (http://goo.gl/zMy5Xz) (08-01-2014).

Dick, W., Carey, L. y Carey, J. O. (2015). The systematic design of instruction (8a ed.). Upper Saddle River: Pearson.

Efland, A. D., Freedman, K. y Stuhr, P. (2003). La educación en el arte posmoderno. Barcelona: Paidós.

Engeström, Y. (1999). Activity theory and individual and social transformation. En Y. Engeström, R. Miettinen y R. Punamäki (Eds.), Perspectives on Activity Theory. Cambridge: Cambridge University Press.

Eisner, E. W. (2002). Ocho importantes condiciones para la enseñanza y el aprendizaje de las artes visuales. Arte, Individuo y Sociedad, 1, pp. 47-55 (http://goo.gl/GjoKWJ).

Filali Marzouki, O., Khalidi Idrissi, M. y Bennani, S. (2017). Effects of Social Constructivist Mobile Learning Environments on Knowledge Acquisition: A MetaAnalysis. International Journal of Interactive Mobile Technologies (iJIM), 11(1). DOI: https://doi.org/10.3991/ijim.v11i1.5982

Freedman, K. (2003). Teaching Visual Culture. Curriculum, Aesthetics and the Social Life of Art. New York: Teachers College Press.

Hernández Hernández, F. (2010). Educación y cultura visual. Barcelona: Octaedro.

Herrington, A. y Herrington, J. (2007). Authentic mobile learning in higher education. En Australian Association for Research in Education (AARE) (Ed.), International Educational Research Conference, 28 de noviembre. Western Australia: Fremantle (http://goo.gl/s2D5sL).

Holmberg, B. (1995). Theory and Practice of Distance Education (2a Ed.). London: Routledge.

Un Marco de referencia para la Enseñanzas Artísticas Visuales en dispositivos Móviles.

Alfredo José Ramón-Verdú, Alfredo Cuervo-Pando, María Gracia Ruiz-llamas. Página 22 de 24 
RED. Revista de Educación a Distancia. Núm. 59, Artíc. 7, 30-04-2019

DOI: http://dx.doi.org/10.6018/red/59/07

Jonassen, D. (1999). Designing Constructivist Learning Environments. En C. Reigeluth (Ed.), Instructional-Design Theories and Models. A New Paradigm of Instructional Theory, 2, pp. 215-239. New York: Routledge.

Jonassen, D. (2000). Toward a Design Theory of Problem Solving. Educational Technology Research and Development, 48(4), pp. 63-85.

Kaptelinin, V. y Nardi, B. A. (2006). Acting with Technology Activity Theory and Interaction Design. Cambridge: MIT.

Koole, M. (2006). The framework for the rational analysis of mobile education (FRAME) model: An evaluation of mobile devices for distance education (Tesis Doctoral). Disponible en el repositorio digital CiteSeerX (http://goo.gl/rJeNiS).

Laurillard, D. (2002). Rethinking University Teaching. A conversational framework for the effective use of learning technologies. London: RoutledgeFalmer.

Laurillard, D. (2007). Pedagogical forms for mobile learning: framing research questions. En N. Pachler (ed.), Mobile learning: towards a research agenda (pp. 153-175). London: WLE Centre, IoE.

Lave, J. y Wenger, E. (1991). Situated Learning: legitimate peripheral participation. Cambridge: Cambridge University Press.

Lombardi, M. M. (2007). Authentic learning for the $21^{\text {st }}$ century: an overview. ELI Papers and Reports. (http://goo.gl/wz3woc).

Moore, M. (1993). Theory of transactional distance. En D. Keegan (Ed.), Theoretical Principles of Distance Education (pp. 20-35). New York: Routledge.

Morrison, D. (2007). E-Learning in higher education: the need to a new pedagogy. En M. Bullen y D. P. Janes (Eds.). Making the transition to e-learning. Strategies and Issues (pp. 104-120). Hershey: Idea Group Inc.

Morrison, G. R., Ross, S. M., Kalman, H. K. y Kemp, J. E. (2013). Designing effective instruction ( $7^{\mathrm{a}}$ ed.). Hoboken, NJ: John Wiley \& Sons, Inc.

Morrow, J. y Holland, J. (2011). Pask and Ma join forces in an elementary mathematics methods course. En IRMA (Ed.), Instructional design: concepts, methodologies, tools, and applications, (pp. 1806-1816). New York: Information Science Reference.

Naismith, L., Lonsdale, P., Vavoula, G. y Sharples, M. (2004). Literature Review in Mobile Technologies and Learning. (http://goo.gl/efKqJO).

Narciss, S. (2008). Feedback Strategies for Interactive Learning Tasks. En J. M. Spector, M. D. Merrill, J. Merriënboer y M. P. Driscoll (Eds.), Handbook of research on educational communications and technology (3a ed.). New York: Lawrence Erlbaum Associates.

Park, Y. (2011). A pedagogical framework for mobile learning: Categorizing educational applications of mobile technologies into four types. The International Review of Research in Open and Distance Learning, 12(2), pp. 78-102 (http://goo.gl/HkvFU).

Pedler, M. (2011). Action learning in practice. Farnham: Gower.

Un Marco de referencia para la Enseñanzas Artísticas Visuales en dispositivos Móviles.

Alfredo José Ramón-Verdú, Alfredo Cuervo-Pando, María Gracia Ruiz-llamas. Página 23 de 24 
RED. Revista de Educación a Distancia. Núm. 59, Artíc. 7, 30-04-2019

DOI: http://dx.doi.org/10.6018/red/59/07

Reigeluth, C. M. (1999). What is instructional-design theory and how is it changing? En C. M. Reigeluth (Ed.), Instructional-design theories and models. Volume II. New York: Routledge.

Reigeluth, C. M. (2012). Instructional Theory and Technology for the New Paradigm of Education. RED, 32. Disponible en http://www.um.es/ead/red/32/reigeluth.pdf

Reigeluth, C. M., Aslam, S. Chen, Z., Dutta, P., Huh, Y., Lee, D., Lin, C., Lu, Y., Min, M. Tan, V. Watson, S. L. y Watson, W. R. (2015). Personalized Integrated Educational System: Technology Functions for the Learner-Centered Paradigm of Education. Journal of Educational Computing Research, 53(3), pp. 459-496.

Revans, R. W. (2011). Action Learning: its origins and nature. En M. Pedler (Ed.), Action learning in practice, (pp. 5-14). Farnham: Gower.

Revans, R.W. (1983). Action Learning: Its Terms and Character. Management Decision, 21(1), pp. 39-50.

Santos, A. (2009). Bringing reality into the classroom. En M. Ally (Ed.), Mobile Learning: transforming the delivery of education and training, (pp. 219-237). Edmonton: AU Press, Athabasca University.

Siemens, G. (2004). Connectivism: A Learning Theory for the Digital Age. International Journal of Instruccional Technology \& Distance Learning, 2(1) (http://goo.gl/YwYWwh) (19-08-2014).

Smaldino, S., Russell, D. Heinich, R. y Molenda, J. (2004). Instructional Technology and Media for Learning (8a ed.). New Jersey: Pearson.

Tinashe Murire, O., Cilliers, L. (2019). Critical Success Factors to Improve the Adoption of Social Media in Teaching and Learning: A Case Study at a Traditional University. International Journal of Interactive Mobile Technologies (iJIM), 13(3). DOI: https://doi.org/10.3991/ijim.v13i03.8485

Traxler, J. (2009). Current State of Mobile Learning. En M. Ally (Ed.), Mobile Learning: transforming the delivery of education and training, (pp. 9-24). Edmonton: AU Press, Athabasca University.

Tripp, S. D. y Bichelmeyer, B. (1990). Rapid Prototyping: An Alternative Instructional Design Strategy. Educational Technology Research \& Development, 38(1), pp. 3144. DOI: $10.1007 / \mathrm{BF} 02298246$

Vygotsky, L. (1978). Mind and society: The development of higher mental processes. En M. Cole, V. John-Steiner, S. Scribner y E. Souberman (Eds.). Cambridge, MA: Harvard University Press.

Wang, M. y Shen, R. (2012). Message design for mobile learning: learning theories, human cognition and design principles. British Journal of Educational Technology, 43, 561-575. DOI: http://dx.doi.org/10.1111/j.1467-8535.2011.01214.x

Watson, W. R., Watson, S. L. y Reigeluth, C. M. (2013). Education 3.0: breaking the mold with technology. Interactive Learning Environments. New York: Routledge. DOI:10.1080/10494820.2013.764322

Un Marco de referencia para la Enseñanzas Artísticas Visuales en dispositivos Móviles.

Alfredo José Ramón-Verdú, Alfredo Cuervo-Pando, María Gracia Ruiz-llamas. Página 24 de 24 
RED. Revista de Educación a Distancia. Núm. 59, Artíc. 7, 30-04-2019

DOI: http://dx.doi.org/10.6018/red/59/07

Willis, J. (2009). A General Set of Procedures for C-ID: R2D2. En J. Willis (Ed.), Constructivist Instructional Design (C-ID): Foundations, Models and Examples, (pp. 313-355). Charlotte: Information Age Publishing.

Yuktirat, Ch., Sindhuphak, A. y Kiddee, K. (2018). M-learning for the Art of Drawing: Informal Learning for a Digital Age. International Journal of Interactive Mobile Technologies (iJIM), 12(5). DOI: https://doi.org/10.3991/ijim.v12i5.9207 